\title{
Effects of Streptokinase on the Development of Rat Cerebral Cortical Cells in Vitro
}

\author{
V. N. Nikandrov and O. N. Zhuk
}

UDC 612.822:576.535:577.17

\begin{abstract}
Translated from Morfologiya, Vol. 128, No. 5, pp. 33-36, September-October, 2005. Original article submitted March 6, 2005, revised version received May 20, 2005.

The aim of the present work was to study the effects of streptokinase (SK) on the ultrastructure of the cellular elements of the cerebral cortex of neonatal rats in vitro. Three series of cultures were used: cultures maintained in DMEM enriched with $15 \%$ fetal calf serum (control 1), some transferred to minimal nutritive medium containing only $0.5 \%$ serum (control 2), and some supplemented with SK (2000 MU/ml) (experimental). Addition of SK to the nutritive medium prevented a decrease in the viability of cells in a mature (14 days) dissociated culture from the neocortex of rat pups aged 1-2 days induced by transfer of the cultures to medium lacking serum proteins. The action of SK on 7-day cultures enhanced the loss of cell viability. Electron microscopy studies of organotypic cultures showed that at this concentration, SK prevented the development of destructive changes of astrocytes, oligodendrocytes, and neurons induced in explants by serum protein deficiency. Neurons showed an abundance of mitochondria, some of which had few cristae. Signs of destruction affected only the nuclei of neurons. After exposure to SK for $48 \mathrm{~h}$, oligodendrocytes were activated (they contained an abundance of myelin-like bodies), with degradation of most astrocytes (surviving examples showing nuclear hyperchromicity). Neurons were resistant to these effects.
\end{abstract}

KEY WORDS: cerebral cortex, tissue culture, cell ultrastructure, streptokinase.

Studies reported by several authors have established the important role of the "plasminogen-plasminogen activator" component in the viability of nerve tissue. This role involves histogenesis, neuritogenesis, the migration of cerebellar granule cells, Schwann cells, etc. [14]. The best studied are the actions of plasminogen activators, especially urokinase and tissue types. However, published data do not cover the whole range. Streptokinase (SK) is an important plasminogen activator - this is a single-chain protein with a molecular weight of $45-55 \mathrm{kDal}$ synthesized by a series of strains of $\beta$-hemolytic streptococci of serogroups $\mathrm{A}, \mathrm{C}$, and $\mathrm{G}$, and is one of the strongest (if not the most powerful) activators of this zymogen. Unlike the plasminogen activators listed above, which are highly specific serine proteases, SK lacks intrinsic proteolytic activity and any kind of hydrolytic activity whatsoever [11].

Laboratory for Regulatory Proteins and Peptides (Director: Professor V. N. Nikandrov), Institute of Physiology, Belarus National Academy of Sciences, Minsk.
Data on the structure and function of this protein have been reviewed in a number of articles [6, 11-13]. One established concept of the function of SK is that of its role in activating plasminogen as a superoxide-converting protein [12]. This view is based on observations that SK has superoxide dismutase-like properties, the suppression of its plasminogen activator activity by a number of superoxide radical scavengers, and the superoxide-generating ability of human and rabbit (but not bovine) plasminogens, which are efficiently activated by SK [12]. The fact that SK not only activates plasminogen, but also converges the superoxide radical (this is of special importance because of the role of active forms of oxygen in the pathology of a whole series of organs and tissues, including neuronal death [5]), and is also produced by opportunistically pathogenic microorganisms constantly present in the bodies of humans and animals, raises the question of the effects of SK on the viability of various body structures.

Traditionally, SK has been regarded as a powerful thrombolytic agent. However, data obtained on the effects of SK on wounds complicated by slow healing, the sup- 
TABLE 1. Effects of Streptokinase on the Viability of Neocortical Cell Cultures from Neonatal Rats

\begin{tabular}{c|c|c|c|c}
\hline $\begin{array}{c}\text { Cultivation period, } \\
\text { days }\end{array}$ & Group No. & Composition of nutritive medium & Cell viability, $\%\left(\bar{p} \pm s_{\bar{p}}\right)$ & Statistical significance \\
\hline \multirow{5}{*}{7} & 1 & DMEM + 15\% FCS & $87.8 \pm 1.6$ & $P_{1-2}<0.01$ \\
\cline { 2 - 5 } & 2 & DMEM + 0.5\% FCS & $82.30 \pm 0.23$ & $P_{1-3}<0.01$ \\
\cline { 2 - 5 } & 3 & DMEM + 0.5\% FCS + SK 2000 MU/ml & $76.4 \pm 1.2$ & $P_{2-3}<0.001$ \\
\hline \multirow{3}{*}{14} & 4 & DMEM + 15\% FCS & $88.13 \pm 0.14$ & $P_{4-5}<0.001$ \\
\cline { 2 - 5 } & 5 & DMEM + 0.5\% FCS & $80.2 \pm 1.4$ & $P_{4-6}<0.1$ \\
\cline { 2 - 5 } & 6 & DMEM + 0.5\% FCS + SK 2000 MU/ml & $89.8 \pm 1.0$ & $P_{5-6}<0.01$ \\
\hline
\end{tabular}

Notes. $\mathrm{FCS}=$ fetal calf serum; $\mathrm{SK}=$ streptokinase.

pression of its production by herpes simplex virus type I and group A viruses in chick embryo fibroblast cultures [9], and the sharp decrease in ATP- and $\mathrm{Ca}^{2+}$-activated proteolysis in PC12 cells [7] lead to the conclusion that SK has direct effects, not mediated via the circulation, on tissues and cells. This aspect of its actions has received little study.

We have previously obtained preliminary data on changes in the structure of cells in nervous tissue on exposure of organotypic cultures of cerebral cortical and sympathetic and spinal ganglion cells to SK [3, 4]. The aim of the present work was to study the effect of SK on the ultrastructure of the cellular elements of the cerebral cortex of neocortical rats in vitro.

\section{MATERIALS AND METHODS}

Studies were performed on organotypic and dissociated cultures of the neocortex from rat pups aged 1-2 days. Experiments used DMEM nutritive medium, fetal calf serum (FCS), trypsin (Sigma, USA), and SK as Streptase (Belmedpreparaty, Belarus). Other reagents were sourced from countries in the CIS.

Organotypic cultures were made by dividing prepared brain tissue into fragments of about $1 \mathrm{~mm}^{3}$, which were placed on collagen-coated glass slides in plastic Petri dishes of diameter $3.5 \mathrm{~cm}$. DMEM medium was enriched with FCS $(15 \%)$ and gentamicin solution was added to $0.01 \%$. The medium was changed after explants had attached and signs of regeneration had appeared ( 2 days in vitro). Three series of cultures were used: those grown in DMEM enriched with $15 \%$ FCS (control 1), those transferred to minimal nutritive medium containing only $0.5 \%$ FCS (control 2), and those containing SK at $2000 \mathrm{MU} / \mathrm{ml}$ (experimental). Plates containing cultures were placed in a Heracell incubator (Switzerland) at a temperature of $37^{\circ} \mathrm{C}$ in an atmosphere containing 5\% $\mathrm{CO}_{2}$. At 24 and $48 \mathrm{~h}$, cultures were fixed for electron microscopy [1]. Ultrathin sec- tions were cut on an LKB III microtome (Sweden), contrasted with lead citrate, and analyzed using a JEM-100B electron microscope (Japan).

Dissociated cultures were prepared by freeing neocortex tissue from its coating layers, mechanical grinding, and treatment with $0.25 \%$ trypsin by standard methods. After inactivation of trypsin, cells were washed twice with DMEM nutritive medium containing 20\% FCS and were then seeded in plastic trays at a rate of 3500 cells per $5 \mathrm{ml}$ of nutritive medium. Medium was replaced with fresh medium twice weekly. Cultures with well developed monolayers were supplemented with SK at the concentration stated above and the serum content in the medium was decreased to $0.5 \%$. Cell viability was determined at 24 and $48 \mathrm{~h}-0.5 \mathrm{ml}$ of cell suspension was diluted five-fold in $0.2 \%$ trypan blue solution (which stains only dead cells) and incubated at $37^{\circ} \mathrm{C}$ for $5 \mathrm{~min}$. Suspensions were placed in a hemocytometer chamber (a Goryaev chamber) and the total number of cells and the number of stained cells were counted in 25 large squares. Further calculation was based on the formula $X=N \times S \times 10000$, where $X$ is the total number of cells per $\mathrm{cm}^{3}$ of suspension, $N$ is the number of cells in the large squares, $S$ is the dilution factor on adding stain, and 10000 is the multiplication constant. The relative content of live cells (not staining with trypan blue) was determined as (total number of cells - number of stained cells)/(total number of cells).

All studies were performed in at least three repeats. The statistical significance of data was assessed using Student's $t$ test.

\section{RESULTS}

Experiments on dissociated cultures established that transfer at 14 days to nutritive medium lacking serum proteins $(0.5 \%$ FCS instead of $15 \%)$ led after $48 \mathrm{~h}$ to a statistically significant reduction in the proportion of viable cells 

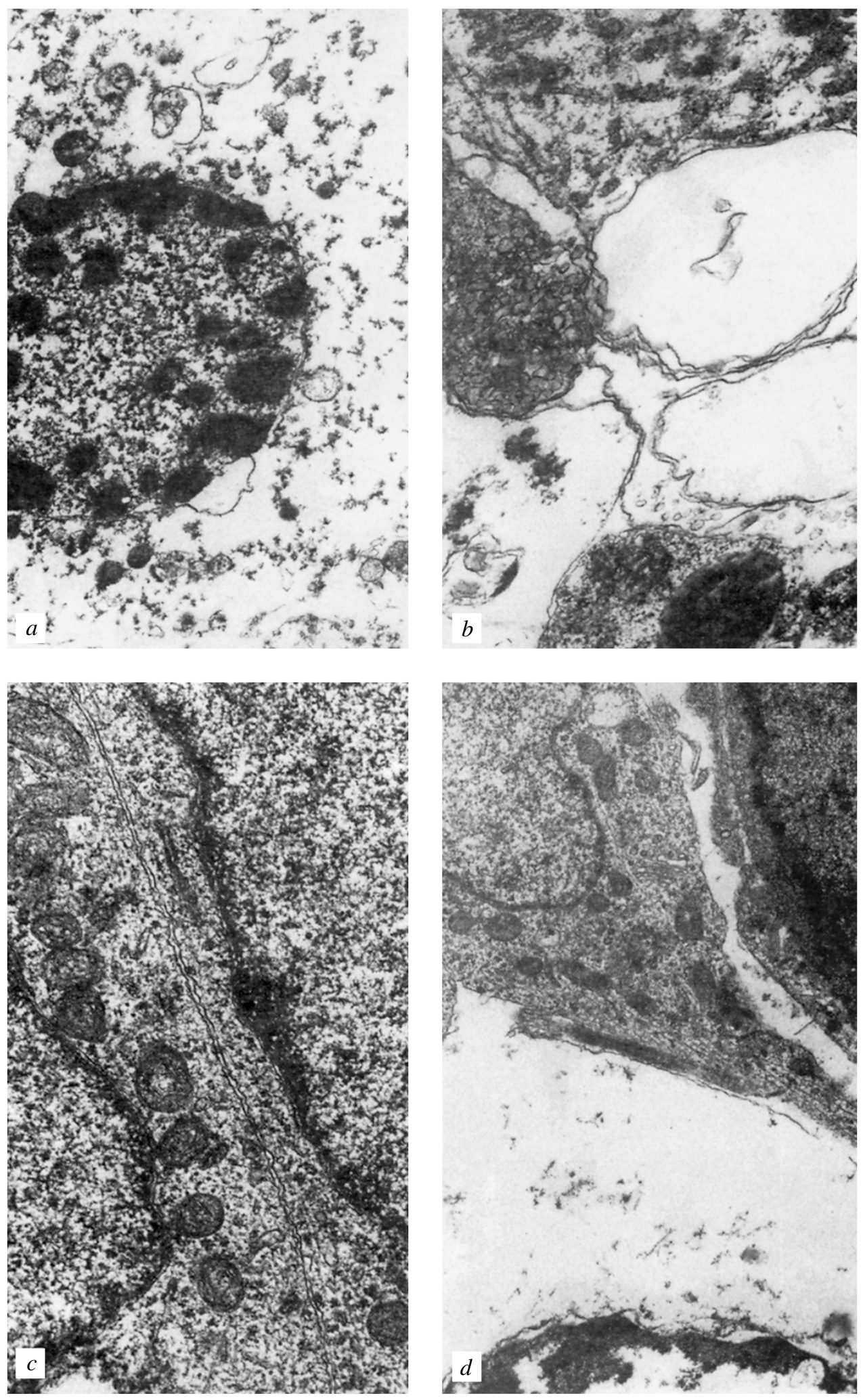

Fig. 1. Effects of streptokinase on the ultrastructure of cells in the cerebral cortex of neonatal rats in vitro. $a$ ) Changes in an astrocyte induced by transfer of the culture to medium depleted of serum proteins (fetal calf serum, FCS, $0.5 \%, 2$ days of exposure); $b$ ) changes in the neuropil on cultivation in nutritive medium containing $0.5 \%$ FCS ( 2 days of exposure); $c$ ) a neuron tightly appressed to another neuron in an explant after $24 \mathrm{~h}$ of exposure in nutritive medium depleted of serum proteins and supplemented with streptokinase; $d$ ) a neuron with adjacent astrocyte and oligodendrocyte in satellite cell positions. Tightly adjacent astrocyte and disintegration of contacts with the oligodendrocyte. Exposure for $24 \mathrm{~h}$ in nutritive medium depleted of serum proteins and supplemented with streptokinase. Magnification: $a, b, d) \times 10,000 ; c) \times 19,000$. 
(Table 1). Addition of SK to this medium maintained this measure at a level similar to that seen in the presence of serum-enriched nutritive medium. Transfer of seven-day cultures to protein-depleted medium was also followed by decreases in the proportion of viable cells. Treatment of these cultures with SK led to a further decrease in the proportion of viable cells.

As shown by electron microscopy results, the structural organization of nervous tissue cells was retained on cultivation of cerebral cortex explants from neonatal rats in medium containing $15 \%$ FCS. Neuron nuclei were located centrally, occupied a significant proportion of the cell volume, had uniformly distributed chromatin, and a smooth and even nuclear coat. The cytoplasm was not significantly altered; organelles were typical and uniformly distributed. Gliocyte structure also retained its characteristic features.

In cultures transferred to medium lacking serum proteins, astrocytes showed chromatin condensation within $24 \mathrm{~h}$, along with the appearance of numerous globules of hyperchromic material showing a preferential localization in the inner nuclear membrane, and formation of blebs of the outer nuclear membrane into the cytoplasm. The cytoplasmic organelles lost their characteristic structure and became vacuolized (Fig. 1, a). Neurons responded with changes in nuclear contours, which formed lobes and invaginations. In the neuropil, there were changes in the shapes of processes, with delamination of the membranes and disappearance of organelles (see Fig. 1, $b$ ).

The destructive changes described above did not appear on cultivation in medium depleted of serum proteins combined with addition of SK (2000 MU/ml). Explant organization was retained, and neurons were tightly appressed to other neurons and astrocytes (see Fig. 1, $c, d$ ). Disintegration of contacts was seen only between neurons and satellite cells (see Fig. 1,c). The structural features of nervous tissue cells - cell and nucleus shape, chromatin distribution - were characteristic of each cell type, and there were no signs of destructive change. The organelles of neurons were well developed and the cytoplasm contained cisterns and Golgi vesicles, polysomes, ribosomes, and endoplasmic reticulum canals. Cells were characterized by an abundance of mitochondria with a moderately electron-dense matrix and clear cristae. Astrocytes were rare after exposure to this medium for $48 \mathrm{~h}$. This provides grounds for suggesting that these cells were subject to the greatest level of damage. Cells containing numerous myelin-like bodies appeared, along with lysosomes and vacuoles. This appeared to result from the absorption of the debris of degenerating cells. Neurons were more resistant to this treatment.

\section{DISCUSSION}

The results presented here support our previous observations: SK can act on cells and tissues directly, without involvement of the blood stream. Fundamentally, this places studies of its biological actions on another base, though from this point of view the effects of SK have received very little study. Previously obtained data on other cell types PC12 pheochromocytoma cells [7] - provided grounds for regarding SK as a regulatory protein. As is evident from the data presented here, the effect of exposure to SK is largely dependent on the types of cellular elements and their state, particularly the degree of tissue maturity. In addition, there are grounds for believing that the effect of SK is a triggering effect and can be directed toward stabilizing cell structures and functions, resulting in an increase in cell viability in unfavorable conditions. However, more prolonged exposure to this plasminogen activator led to clear destructive changes in cells. The impression was that the most sensitive cells in the cerebral cortex were astrocytes, while neurons were the most resistant. This is not to say that they were completely resistant. As shown by electrophysiological studies [10], superfusion of pontobulbospinal preparations from rat brains with SK solutions led to decreases by $50-55 \%$ in the frequency with which respiratory volleys were generated, with decreases by $15-20 \%$ in the amplitude of low- and intermediate-frequency discharge peaks. Thus, changes in neurons also occurred.

An important question is that of the pathways (mechanisms) of action of SK on cells. SK is a xenogenic protein; there are no data on the presence of SK receptors on cell membranes, though this possibility cannot be completely excluded. On the other hand, its action via the plasminogen activator pathway (plasminogen receptors are found on many cells) seems very unlikely. SK was used at quite high concentrations in our experiments - of the order of $10^{-6} \mathrm{M}$. Active plasmin is known not to form in the presence of excess SK $[11,13]$. It is important to note that in our experiments with SK on cultures of sympathoblasts and spinal ganglia did not demonstrate the appearance of fibrinolytic (plasmin) activity [8], though enzymatic analysis provided evidence of virtually undegraded SK in these cultures. The action of SK may also be mediated via the superoxide-converting pathway. However, the molecular-cellular aspects of the action of this protein are far from entirely clear.

Finally, the data obtained here provide an avenue for studying the actions of SK on nervous tissue cells with different structures, functions, and metabolic states. These studies are important from the basic science point of view and in terms of applied aspects. Thus, in vitro studies of the effects of SK on the structure of spinal ganglia from adult rats showed cell destruction (affecting neurons and glial cells), along with dyscomplexation of the extracellular matrix [2].

\section{REFERENCES}

1. N. N. Bogolepov, Methods for Electron Microscope Studies of the Brain [in Russian], Sechenov First Moscow Medical Institute Press, Moscow (1976). 
2. O. I. Volodkovich, N. A. Dolgova, V. S. Lukashevich, and V. N. Nikandrov, "Effects of streptokinase in vitro on cell structure and lactate dehydrogenase activity in the spinal ganglia of rats," in: Questions of the Integration of Functions in Physiology and Medicine [in Russian], PChUP Biznesofset Press, Minsk (2004), pp. 67-69.

3. O. N. Zhuk and V. K. Nikandrov, "Structural changes in cells in organotypic cultures of the cerebral cortex of neonatal rats exposed to streptokinase," in: Proceedings of the Jubilee Conference for the 50th Anniversary of the Institute of Physiology of the Belarus National Academy of Sciences [in Russian], Minsk (2003), pp. 57-58.

4. O. N. Zhuk, E. F. Polukoshko, and V. N. Nikandrov, "The effects of streptokinase on viability, development, and the structural-functional organization of cells in the cerebral cortex and some peripheral ganglia in neonatal rats in tissue culture," in: Questions of the Integration of Functions in Physiology and Medicine [in Russian], PChUP Biznesofset Press, Minsk (2004), pp. 133-134.

5. I. A. Zavalishin and M. N. Zakharova, "Neuron death - a cardinal question in neurology and psychiatry," Vestn. Ros, Akad. Med. Nauk, No. 1, 28-33 (1999).

6. V. N. Nikandrov, "Structural organization of the streptokinase molecule," Bioorg. Khim., 20, No. 2, 169-181 (1994).

7. V. N. Nikandrov, G. P. Petrusenko, and R. I. Gronskaya, "The state of ATP- and $\mathrm{Ca}^{2+}$-dependent proteolysis in $\mathrm{PC} 12$ pheochromocytoma cells exposed to streptokinase and nerve growth factor,"
Izv. Nar. Akad. Nauk Belarusi: Ser. Med.-Biol. Nauk, No. 4, 84-87 (2003).

8. V. N. Nikandrov, G. P. Petrusenko, N. N. Zhuk, et al., "Effects of components of pericellular proteolysis on cells in nervous tissue," in: Achievements of Belarus Medical Sciences [in Russian], Retsenzir. Nauchno-prakt. Ezhegodnik, Minsk (2002), No. 7, 49-50.

9. V. N. Nikandrov and N. S. Pyzova, "Regulatory proteins: functional properties of the molecules and the mechanisms of their biological actions," Izv. Nar. Akad. Nauk Belarusi: Ser. Med.-Biol. Nauk, No. 3, 75-89 (2003).

10. V. N. Nikandrov, V. F. Pyatin, S. A. Alekseeva, et al., "Modulation of central respiratory activity using plasminogen, streptokinase, and their complexes with pyruvate kinase," Izv. Nar. Akad. Nauk Belarusi: Ser. Med.-Biol. Nauk, No. 2, 40-43 (2003).

11. F. J. Castellino, A unique enzyme-protein substrate modifier reaction: plasmin streptokinase interaction," Trends Int. Biochem. Soc., No. 1, 1-5 (1979).

12. V. N. Nikandrov, "On the plasminogen-activating function of streptokinase," Int. J. Biochem., 24, No. 1, 43057 (1992).

13. K. N. N. Reddy, "Streptokinase - biochemistry and clinical applications," Enzyme, 40, No. 1, 79-89 (1988).

14. D. E. Rosenblatt, C. Cotman, M. Nieto-Sampedro, et al., "Interaction of a protease inhibitor produced by astrocytes that is structurally and functionally homologous to human protease nexin-I," Brain Res., 415, 40-48 (1987). 